\title{
Questions and responses in Lao
}

\author{
N.J. Enfield ${ }^{\mathrm{a}, \mathrm{b}, \mathrm{c}, *}$ \\ ${ }^{a}$ Max Planck Institute for Psycholinguistics, PB 310, 6500 AH Nijmegen, The Netherlands \\ ${ }^{\mathrm{b}}$ Radboud University Nijmegen, PB 9102, 6500 HC, The Netherlands \\ ${ }^{\mathrm{C}}$ Donders Institute for Brain, Cognition, and Behaviour, PB 9102, $6500 \mathrm{HC}$, The Netherlands
}

\section{A R T I C L E I N F O}

\section{Article history:}

Received 29 March 2010

Accepted 2 April 2010

\section{Keywords:}

Lao language

Questions

Polar (yes/no) questions

Content $(\mathrm{WH})$ questions

Conversation

\begin{abstract}
A B S T R A C T
This paper surveys the structure of questions and their responses in Lao, a Southwestern Tai language spoken in Laos, Thailand, and Cambodia. Data are from video-recordings of naturally occurring conversation in Vientiane, Laos. An outline of the lexico-grammatical options for formulating questions describes content ('WH') questions and polar ('yes/no') questions. The content question forms are from a set of indefinite pronouns. The WHAT, WHERE, and WHO categories have higher token frequency than the other categories. Polar questions are mostly formed by the addition of different turn-final markers, with different meanings. 'Declarative questions' (i.e., polar questions which are formally identical to statements) are common. An examination of the interactional functions of questions in the data show asymmetries between polar and content questions, with content questions used mostly for requesting information, while polar questions are also widely used for requesting confirmation, among other things. There is discussion of the kinds of responses that are appropriate or preferred given certain types of question. Alongside discussion of numerous examples, the paper provides quantitative data on the frequencies of various patterns in questions and responses. These data form part of a large-scale, ten-language coding study.

(c) 2010 Elsevier B.V. All rights reserved.
\end{abstract}

\section{Introduction}

This paper presents a first survey of questions and responses in Lao conversation. ${ }^{1}$. While something is known of the grammatical forms and semantic structure of interrogatives of various types in Lao (Enfield, 2007), the present paper offers a more systematic view, with quantitative data, not only of questions but of the relations between questions and the responses they engender.

The description and analyses are based on data used for the ten-language comparative coding project described in the introduction to this special issue. The data are video recordings of maximally informal conversation between relatives, neighbors and friends in their home settings, in several villages of Vientiane Municipality, Laos. I draw specifically on a corpus of 351 question drawn from 8 separate recordings (see appendix). Each selection ranges from 6 min to 16 min in length. Of these, 3 recordings were dyadic (32 min; i.e., $41 \%$ of the total), the rest involved three or more people. All were face-to-face video recordings of people in maximally informal conversational settings, not institutional, task-based, or otherwise pre-arranged. These were people going about their daily business. They gave permission to make the recordings and to analyze and publicize the results.

\footnotetext{
* Correspondence address: Max Planck Institute for Psycholinguistics, PB 310, 6500 AH Nijmegen, The Netherlands. Tel.: +31 24 3521 275; fax: +31243521213

E-mail address: Nick.Enfield@mpi.nl.

${ }^{1}$ Lao is a Southwestern Tai language, spoken in Laos, Thailand, and Cambodia. It is the national language of Laos. For detailed information on the language and its structures, including the transcription and glossing conventions used here, see Enfield (2007).
} 
Total time of conversation yielding 351 questions was $78 \mathrm{~min}$. This is an average of about 1 question every $13 \mathrm{~s}$, showing how ubiquitous questions are in everyday, turn-by-turn talk. Note that 42 cases ( $12 \%$ of the full set of 351 ) are not counted as questions for purposes of the ten-language comparative study. The introduction to this special issue describes the criteria that were used for identifying questions in formal and functional terms. These included formally interrogative utterances which did not function as questions in any meaningful way: e.g., rhetorical questions like 'How on earth can they eat food so spicy?', and exclamations like 'Oh well, what can you do?'. The total number of cases included in this study is 309.

\section{Results}

\subsection{Lexico-grammatical options for question formulation}

Lao has two major categories of question, distinguishable in terms of underlying information structure. Polar questions present an entire proposition (e.g., 'John washed the dishes'), and ask as to the truth or falsity of that proposition (Did John wash the dishes?). A variant is the alternative question, which effectively presents two propositions to choose from; Did John wash the dishes or dry them? By contrast, content (or 'WH') questions presuppose the truth of some elements of a proposition and ask as to the reference of a component whose identity is unknown. So, Who washed the dishes? presupposes that somebody washed the dishes, but leaves open the identity of this person. In informational terms, the aim of the question is to elicit this missing information.

In line with what is known for other languages (see other papers in this special issue), polar questions are more frequent in conversation than content questions. Polar questions account for nearly three quarters of all questions in the Lao corpus $(223 / 309=72 \%)$. Alternative questions are rare $(2 / 309<1 \%)$.

This section describes the basic lexico-grammatical resources for formulation of questions in Lao. For further detail, see Chapters 4 and 5 of Enfield (2007).

\subsubsection{Content questions ('WH questions')}

There are three independent indefinite pronouns which may function as content question words. These are listed in (1).

$$
\begin{aligned}
& \text { Independent indefinite pronouns } \\
& \begin{array}{ll}
\text { nang3 } & \text { 'what', 'anything', 'something' (INDEF.INAN) } \\
\text { phaj3 } & \text { 'who', 'anyone', 'someone' (INDEF.HUM) } \\
\text { saj3 } & \text { 'where', 'anywhere', 'somewhere' (INDEF.LOC) }
\end{array}
\end{aligned}
$$

In some contexts, these are interpreted as question words, as in the examples (2-4).

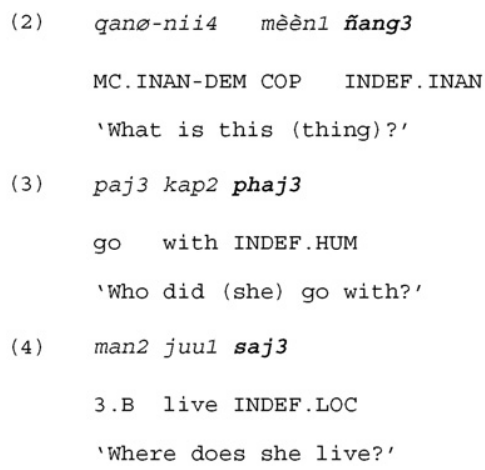

In other contexts they are interpreted as indefinite pronouns, as in (5-6) (see also example (15), below).

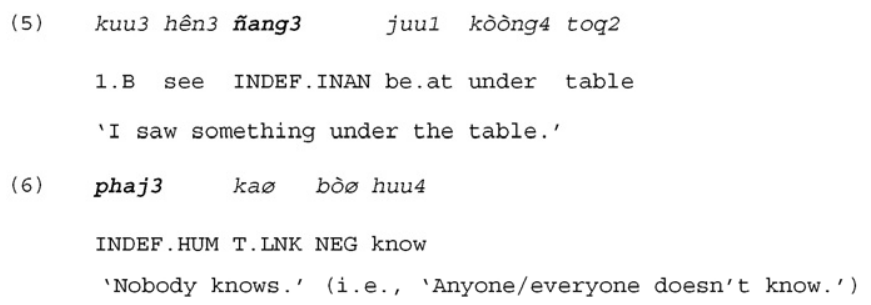


There is a generic, dependent indefinite pronoun, which shows the same possible interpretation. This is daj3, a postposed modifier of any nominal head (usually a classifier), which can mean 'which N?', 'any N', or 'some N'.

(7) caw4 mak1 khan2 daj3

2SG.P like CLF.VEHICLE INDEF

'Which one (vehicle) do you like?'

This has an all-purpose function in deriving a wide range of content question forms, along the lines 'in which way', 'at which place', 'in which manner', and so on.

Another indefinite nominal modifier is cak2, which is pre-posed to a nominal head, to mean either interrogative 'how many' as in example (8) or indefinite 'some/any amount' as in example (9).

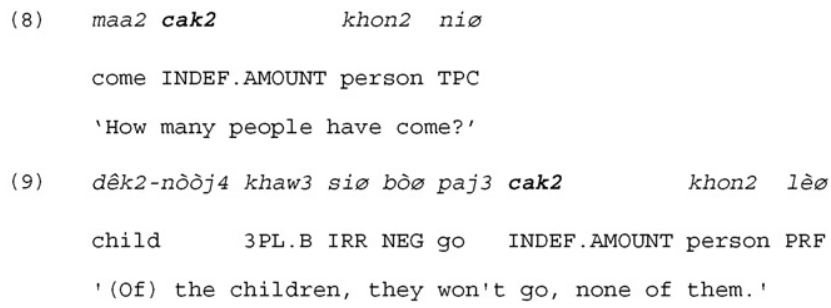

Whether the indefinite pronominal forms function as indefinites ('some, any, whichever') or as interrogatives ('what?', 'who?', 'which?') depends on grammatical and information structural context. I argue that it is not necessary to propose distinct interrogative meanings for them (Enfield, 2007:86; cf. Wierzbicka, 1980). Because indefinite reference is semantically simpler than interrogative reference, it may be incorporated within the more complex semantics of 'WH words'. So, 'Who?' means something like ‘someone, I don't know who', incorporating reference to 'someone' (cf. Wierzbicka, 1996). The same applies to 'something/what', 'some/which', 'somewhere/where', etc. Whichever interpretation indefinite pronominal expressions are given, they always convey at least the invariant meaning 'INDEF' (i.e., 'some/any X'). Listeners use pragmatic implicature (Grice, 1975; Levinson, 1983; Levinson, 2000; Sperber and Wilson, 1995) to yield interrogative utterance-level meaning by enriching indefinite sentence-level semantics. Another argument in favor of the idea that an indefinite meaning is more basic than an interrogative one is that there are few if any structural contexts in which the indefinite pronouns listed above must be read as interrogative, while there are multiple structural contexts in which only indefinite readings are possible, and interrogative readings are unavailable. In sum, where indefinite pronouns may have two interpretations (interrogative 'who?', 'what?', 'which N'? versus indefinite 'someone', 'something', 'some N'), discourse context determines the right interpretation. See Enfield (2007) for extended arguments in support of this.

Two structures may be used for asking 'why?'. ${ }^{2}$ One involves the expression pên $3 \tilde{n} a n g 3$, a combination of the copula pên3 and the indefinite inanimate pronoun ñang3-literally ‘to be what?'-in sentence-initial position. This is illustrated in example (10).

(10)

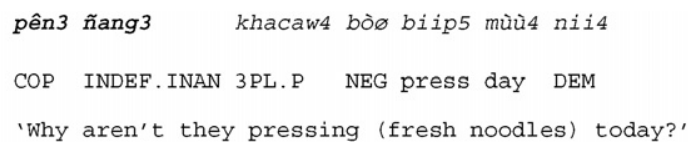

A second way of asking 'why?' involves the verb khùù2 'to be like' in preverbal position, as in examples (11-12).

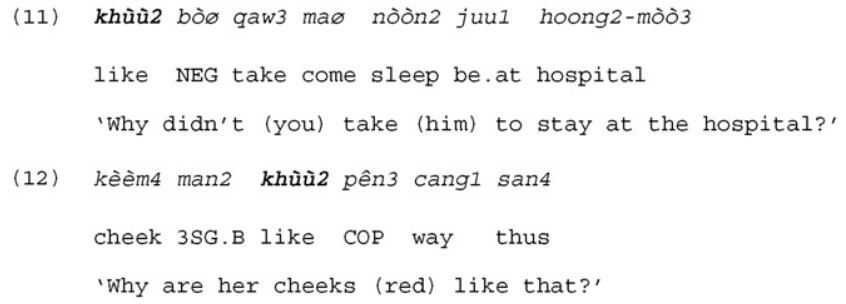

\footnotetext{
${ }^{2}$ There is a subtle difference in meaning between these two. The form pên 3 ñang3 is neutral, while the form khùù asks why something is so, while conveying a subtle sense that it should not be so.
} 


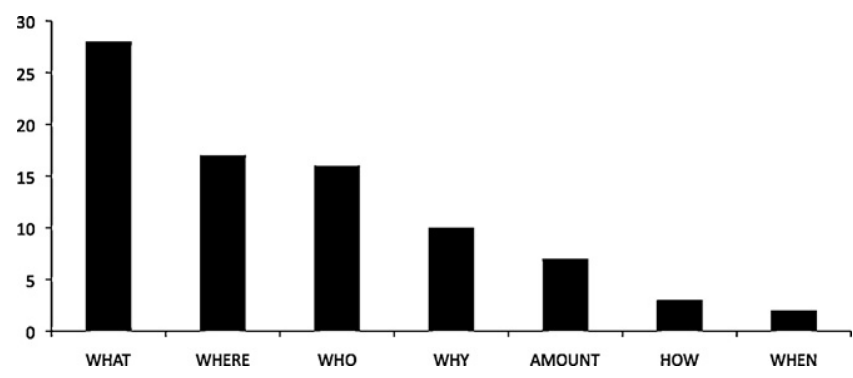

Fig. 1. Frequencies (as $n / 84$ ) of different categories across content questions in Lao.

These two strategies can be combined, as in example (13).

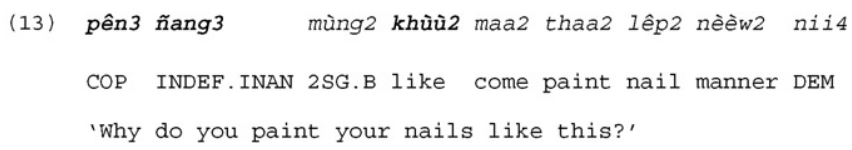

The relative raw frequencies of these categories in the Lao corpus are shown in Fig. 1.

\subsubsection{Polar questions}

Polar questions are generally marked by the addition of one (or more) of a set of final particles (see chapter 4 of Enfield, 2007). I shall refer to them in the context of questions as turn-final question markers. There is no other way to explicitly mark a polar question: i.e., no inversion of the kind used in English, and no use of intonation (e.g., rising pitch) as a reliable marker of questions. ${ }^{3}$ When a turn-final question marker is present, this marker often has rising intonation. Turn-final markers appear to have greater freedom in this lexical tone language to show the kind of prosodic variation observes in languages like English, i.e., turn-final rising intonation (see Enfield, 2007:72). But if there is no turn-final marker, it is not possible to use rising intonation as the sole formal signal that the utterance is a question. Lao is a tone language, and the kinds of item that would occur turn-finally in the absence of a turn-final particle (i.e., open class items like nouns and verbs or grammatical markers like aspectual-modals) must maintain their randomly assigned lexical tone, whether that tone happens to be rising, falling, or level in pitch.

Most polar questions $(186 / 223=83 \%$ ) are explicitly marked by a turn-final marker. There are several such markers in Lao. They each make different semantic distinctions. A set of nearly 30 sentence-final particles is described in (Enfield, 2007), expanding on Crisfield (1974). The particles are divided into four classes: interrogative, factive, imperative, and other. We are concerned here with the polar interrogative markers, listed in (14). ${ }^{4}$

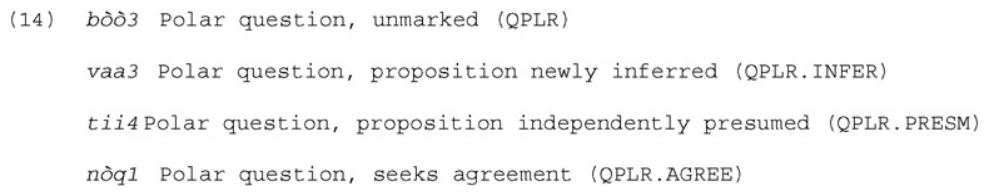

A description of the meanings and functions of each marker may be found in Chapter 4 of Enfield (2007). I supply just a few illustrative examples here.

Examples (15-17) illustrate the turn-final marker bòò, a 'general polar question marker'. ${ }^{5}$

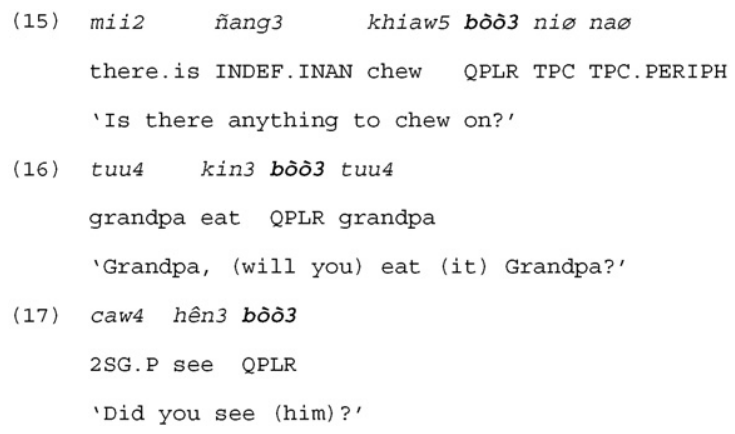

\footnotetext{
3 This does not mean that every question has to be formally marked. Context is often enough (see section 2.1.3, below).

4 Some turn-final question markers are appended to content questions. These are kòq2 (asks for information currently presupposed; Q.PREsuP), hùù2 (emphatic, shows mild annoyance at not knowing; Q.EMPH), nòò (wondering, 'out-loud' question to oneself; Q.WNDR), buq2 (rhetorical question, speaker does not know; Q.unkN). For an example, see (33) below.

${ }^{5}$ What is referred to here as a 'turn-final' marker happens not to be in turn-final position in these two examples. In each case, the marker represents a point of possible turn completion.
} 
There is occasional use of a 'tag question' type structure, in which the combination of a copula mèèn 1 'to be the case' and an interrogative marker such as bò̀ 3 are appended to the end of a sentence. An example is shown in (18).

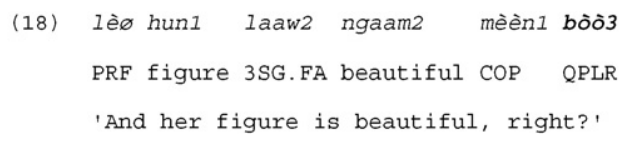

The particle bò̀ 3 provides the closest thing to an unmarked way of forming a polar question in Lao. This means that of the available alternatives, bòò is the least biased toward a particular answer. Alternatives to bòò are marked in the sense that they add more specific semantics to the kind of question conveyed, giving information about the speaker's stance toward the proposition in question. An example is vaa3, which has the same core polar question semantics as bòò, and specifies in addition that the speaker has some current reason to infer that the proposition in question is, in fact, true. In an example, an older man arrives in a village from the city in a pickup truck, and a younger man, a neighbor, drops by to talk to the older man. The younger man knows that another man named Loy has been in the city with his children. He notices Loy's children playing nearby and thus infers that Loy himself has probably also come back to the village with them. Accordingly, he uses vaa3 in phrasing a polar question which checks on the truth of this newly inferred idea. This is shown in example (19).

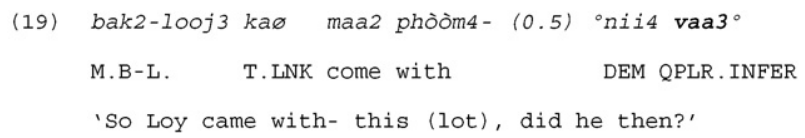

In another example, a man is talking about the virtues of a certain herbal medicine. At one point, he remarks that his stomach problems have eased. One of his addressees infers that this is due to the medicine, and uses vaa3 in phrasing the polar question which seeks confirmation. This is shown in example (20).

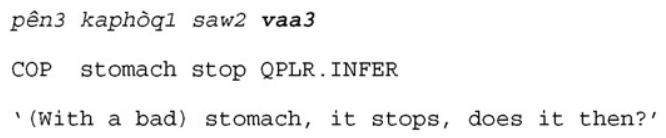

Further examples of different kinds of polar question particles appear in sections below.

\subsection{3. 'Declarative questions'}

It seems that in all languages it is possible to effectively ask a polar question yet without using any formal marking dedicated to that function. This relies entirely on pragmatic interpretation. A standard way to do this is to exploit default asymmetries between speaker and addressee in epistemic access to information. A basic condition for a question to be appropriate is that the speaker should lack some information which the addressee is presumed to have (Searle, 1969). If a statement is in the second person-i.e., if the proposition is about something in the addressee's domain of authority-then this in itself can bring the appropriate asymmetry into the common ground. For instance, imagine that we are talking about how to get to the restaurant, and you start talking about the bicycle route. I might infer from this that you don't have your car today. Since you are the one to know better than I whether this is true, I may simply look at you and say You didn't bring your car today. In turn, you may treat this as a question, answering, simply, No.

Fewer than 20\% (37/223) of the Lao polar questions had no explicit marking showing that they were a question. None of these had rising intonation that could be analyzed as reliably signaling question-hood. Because Lao is a tone language, lexical items must be pronounced with their lexically dedicated pitch contour, and cannot be overridden with 'rising tone'.

Examples of questions which have no formal marking (given in bold face, and with their responses also supplied, showing that these formally declarative structures are treated as questions) are given in (21-22).

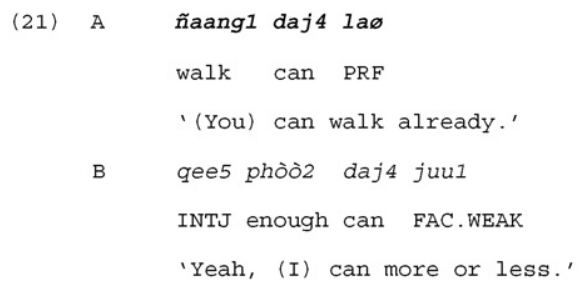




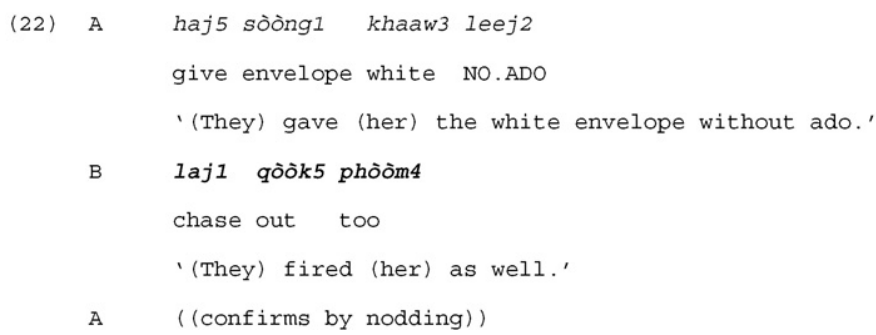

In both of these examples, the epistemic authority of the addressee is given by common ground in the speech event. In (21), Speaker B's healing process from an injury is the current topic of conversation. He is the one who knows better about what is possible for him in his present condition. In (22), Speaker A is telling a story which is entirely new to Speaker B.

\subsection{Range of social actions performed by questions}

Questions are employed by speakers to perform different social actions. Distinctions between types of question based on information structure (e.g., polar versus content questions) cross-cut the kinds of social action they may be used for. For example, in English, What are you doing? could be a request for information (gloss = 'What you're doing looks interesting, what is it?'), or it could be a complaint (gloss = 'What the hell are you doing?'), and may be used in the interests of other interactional goals at the same time. In the full set of Lao questions used in this coding study, just over $40 \%$ are being used to request information $(129 / 309=42 \%)$. For example, in $(23)$, Speaker A is on a visit to Speaker B. Speaker B, who has been sitting down since Speaker A arrived, has recently had an accident and had to stay in bed for several days. A asks whether B is yet able to walk. Although the question may be serving other functions as well, its primary goal here is to find out whether he can walk or not.

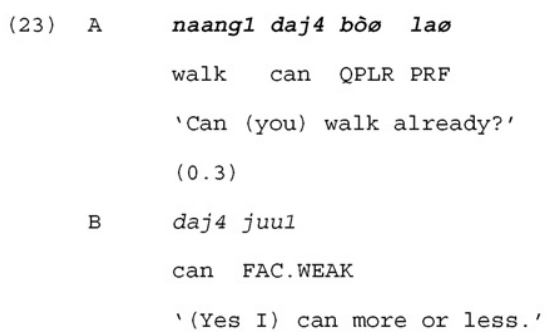

In another example, Speaker A announces that she received a phone call from a woman the evening before. Speaker B requests confirmation, and then asks 'What did she say?', primarily to extract information. This is shown in example (24).

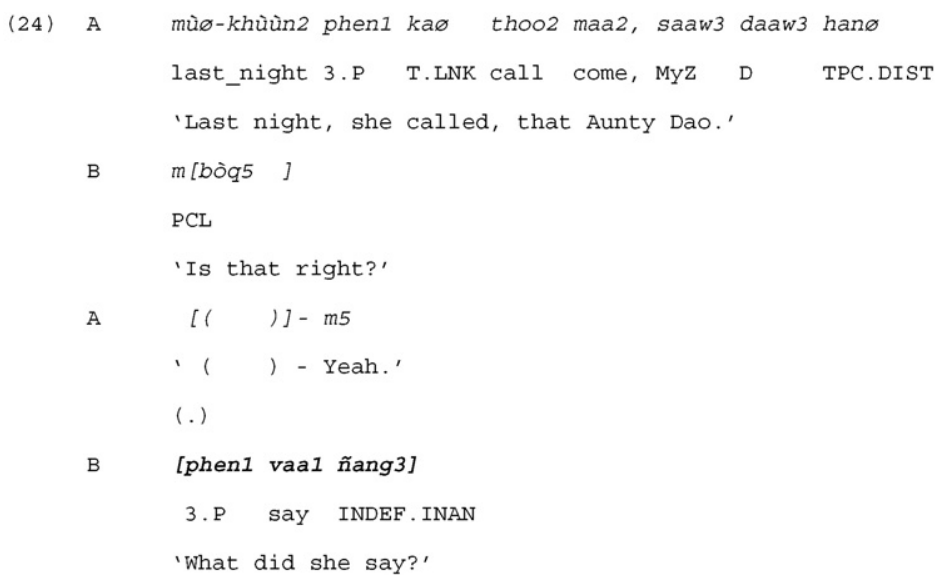

In another example, Speaker A has been gossiping to Speaker B about her boss, who-it is alleged-goes out at night and gets blind drunk. Speaker B asks where the boss has been going. This question, primarily designed to get information, is shown in example (25). 


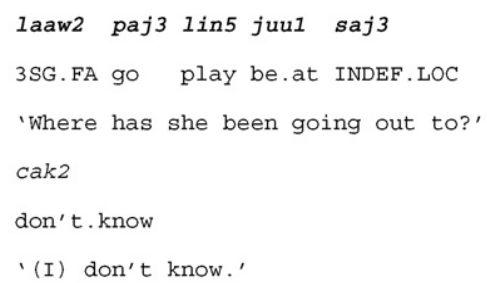

Over a quarter of the questions in the Lao corpus function as requests for confirmation ( $87 / 309=28 \%$ ). (Note that of these, all are polar questions, which means that nearly $40 \%$ of polar questions-87/223-function as requests for confirmation.) An example is given in (26). Here, Speakers A and B are talking about the location of a certain village, and about which routes would be further to go in order to get there. At the beginning of the example, Speaker A asserts that the place in question is far away. Speaker B challenges this directly, stating the opposite, then adding that there is a short cut. This addition of information suggests to A that perhaps it is not far after all. Speaker A thus requests confirmation in the next line, using the polar question marker vaa3 to mark his recent inference of the likely truth of the proposition. It is confirmed by B with a 'head toss' (sharp upward movement of the head; see below).

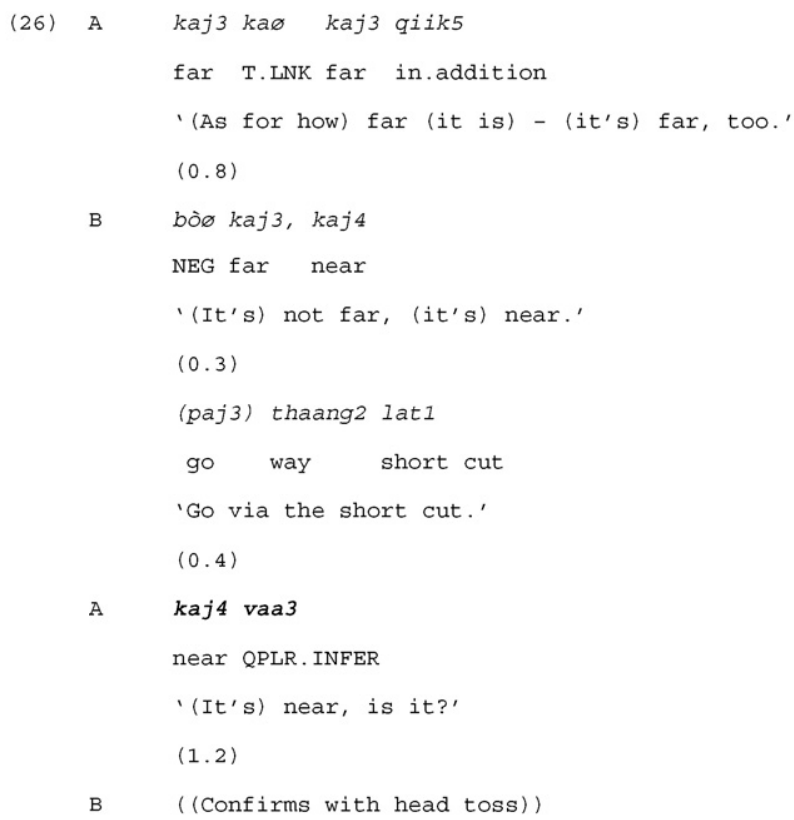

In another example, Speaker A works at an embassy in Vientiane, and is talking about a colleague who was shirking her responsibilities at work. One day, the colleague was found to be absent from her work place without reason. The next day, the colleague was called to the embassy, where, Speaker A says, she was 'given the white envelope'. This is an indirect way of saying that someone was fired-i.e., that they were handed an envelope in which they would find a letter of release, and typically also their severance pay. Because the idiom is indirect, Speaker B asks two questions to clarify that these two probable components of the scenario are in fact the case, i.e., 1. that the colleague was fired, and 2 . that she was given severance money. Note that Speaker B's requests for confirmation are both polar questions, the first in declarative form, the second with the 'tag' structure involving the copula mè̀n1. This is shown in example (27).

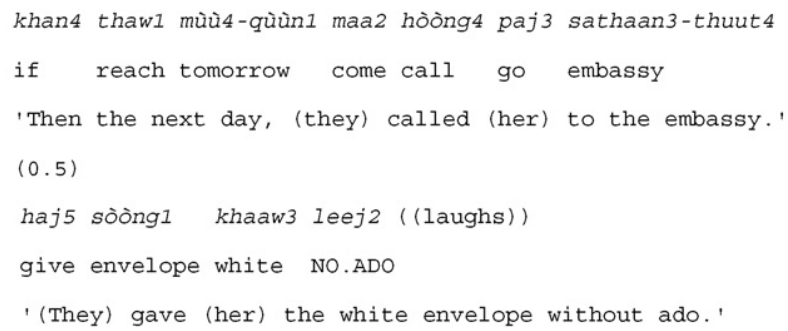


$(0.2)$

laj1 qòòk5 phòòm4

chase out too

'(They) fired (her) too.'

A

((confirms by nodding))

(0.5)

B

haj5 ngen2 mèèn1 bòò3=

give money COP QPLR

'(They) gave (her the) money, right?'

$=m m 4$

'Yeah.' ((confirms with head toss))

Another category of reasonable size $(49 / 309=16 \%)$ is made up of those questions functioning as 'assessments', i.e., as ways of expressing one's appraisal or evaluation of the properties of something (cf. English Isn't this delicious?). An example is in (28), where Speaker A has just been handed some herbal medicine by Speaker B, and he is sniffing it. His assessment that the medicine is fragrant is marked with the agreement-seeking particle nòq1, which is more or less dedicated to this function, i.e., inviting agreement from another speaker on an assessment.

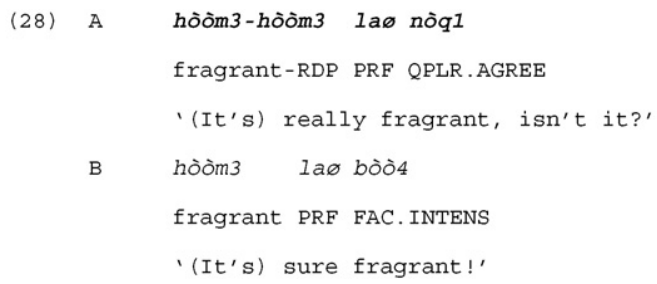

In another example, Speaker A is gossiping to Speaker B about a colleague, conveying in various ways the improper nature of the young woman's behavior in the workplace. In the first few lines of example (29), Speaker A is describing the woman's inappropriate ways of dressing, which includes regularly changing her hair color and the color of her nails. Speaker B makes an assessment that this is 'weird', appending the agreement-inviting marker nòq1, as also seen in example (28).

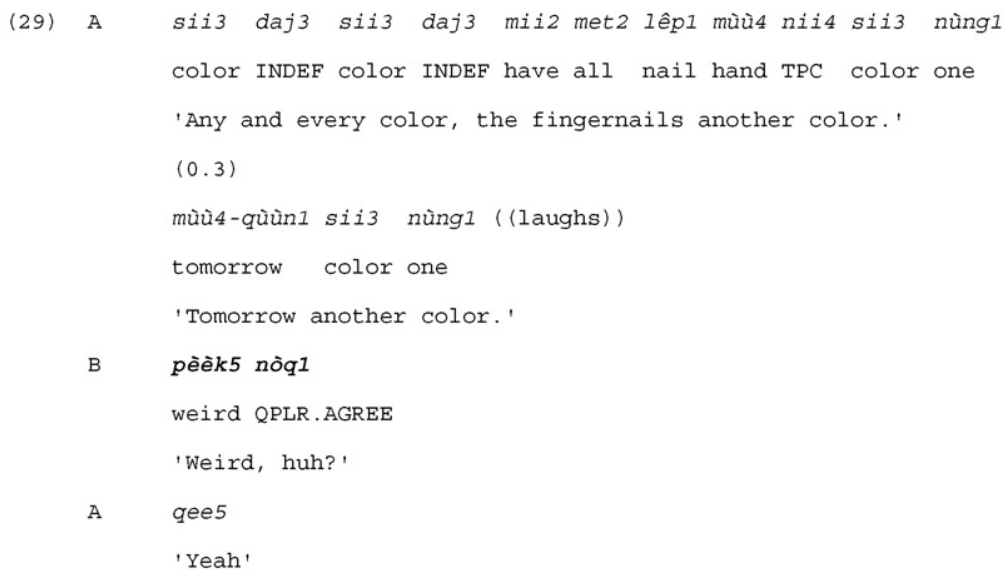

Note that the questions which function to convey assessments in this way are all polar questions (which means that $22 \%$ of polar questions-49/223-function to convey assessments).

A smaller functional category is formed by questions that initiate repair (e.g., What? when one has not clearly heard what was just said). These make up just $6 \%$ of the total set (17/309), with a more or less even division between polar and content questions having this function (7 versus 10 instances, respectively). An example is shown in (30), in which Speaker A is discussing his plan to drive to neighboring villages in order find a particular type of rare fish trap. In trying to convey that he plans to donate one of these traps to the Fishery Department in the city as a kind of exhibit or showpiece, he can't seem to find 
the right word. In the first line of example (30), he cuts off his speech where the word would have come, and he then simply continues on in the next line, leaving a gap. Speaker B offers a candidate for what Speaker A might have wanted to say in that first line. He suggests the word thilanùk1 'souvenir', marking this with a turn-final question marker tii4 (whose general function is to mark that the speaker presumes the proposition is right, but is checking to be sure). This elicits confirmation from Speaker A that this is indeed an appropriate gloss for what he wanted to say.

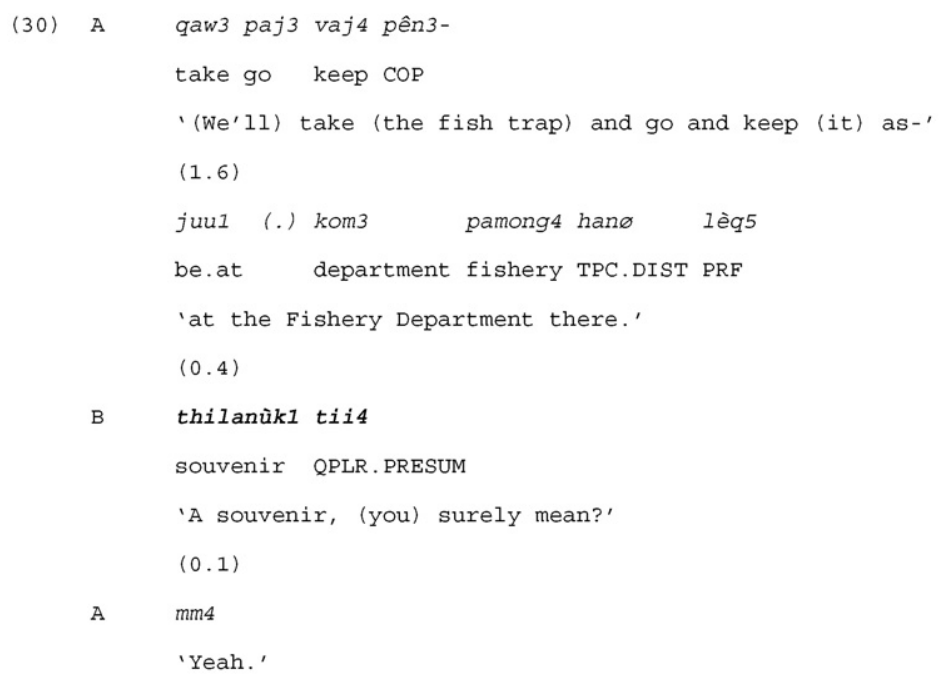

In another example, we see a content question-using phaj3 'who?'-being used for initiation of repair. In the first line of example (31), Speaker A's reference to a person is semantically very general (literally, 'that person'), and Speaker B evidently doesn't have the required information to know who Speaker A is talking about. Speaker B initiates repair with the indefinite human pronoun phaj3, here to be interpreted as 'who?'. This elicits a more specific statement from Speaker A of the person reference, now as 'the person who I used to work with'. This is shown in example (31).

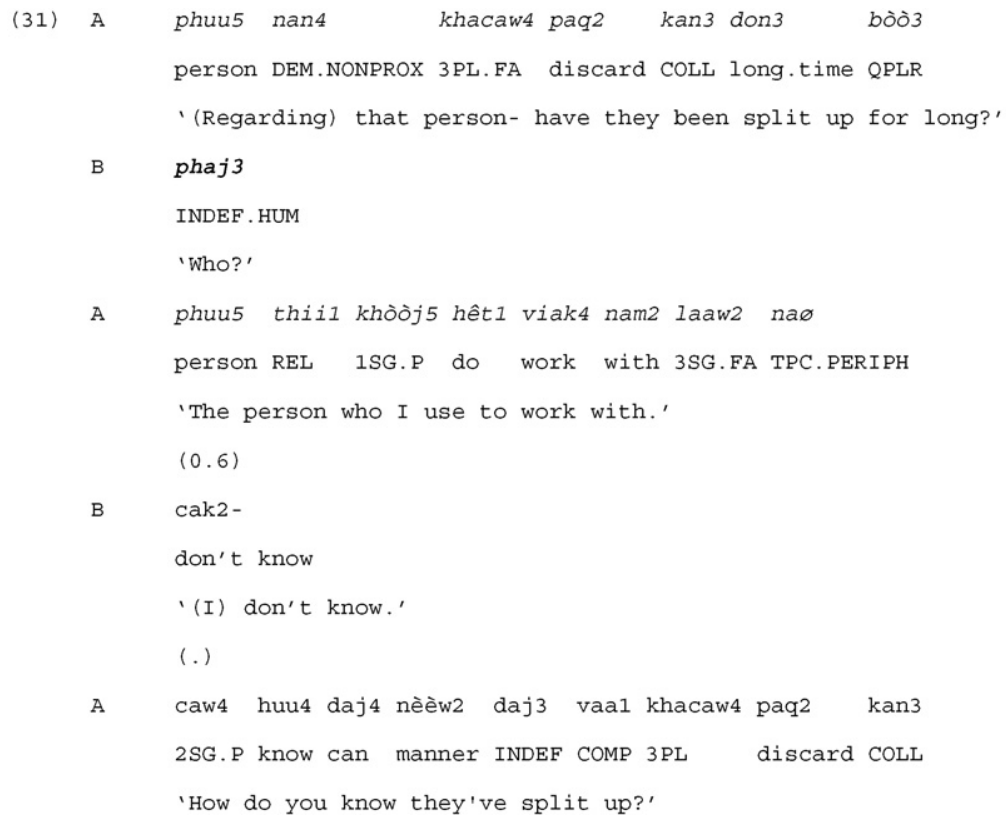

Fig. 2 shows a breakdown of all questions by their function in context.

Fig. 2 lumps polar and content questions together. If we pull these apart, we see that content questions are mostly dedicated to seeking information or initiating repair, and they are not used for requesting confirmation, or doing assessments. This is shown in Fig. 3. 


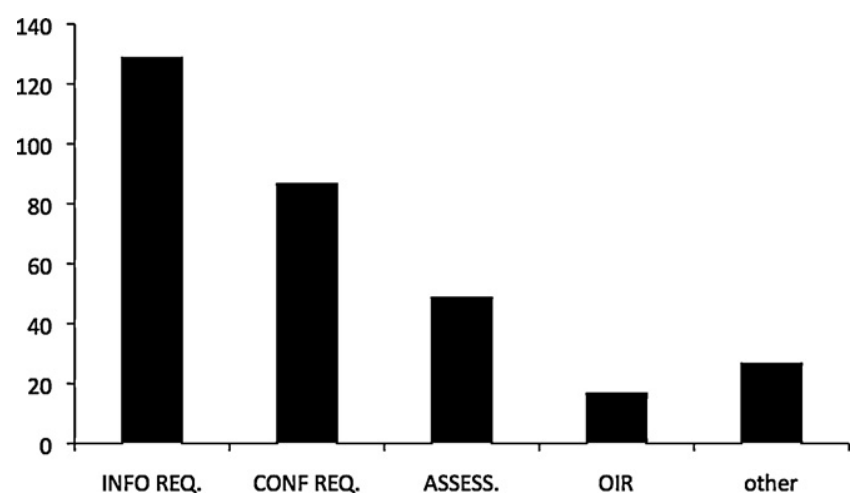

Fig. 2. Frequencies (as n/309) of different functions across all questions. Here and below: 'OIR' stands for 'Other Initiation of Repair'.

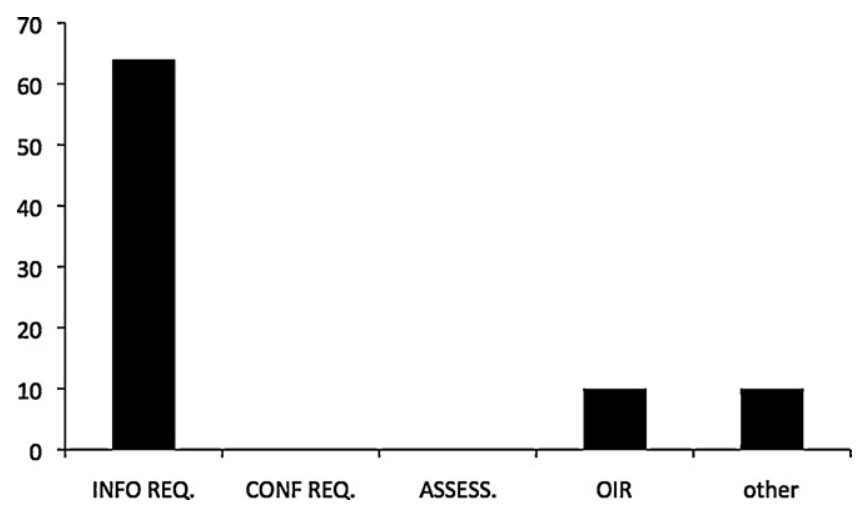

Fig. 3. Frequencies (as $n / 84$ ) of different functions across content questions.

Polar questions, by contrast, show a broader range of functions, with a considerable number of requests for confirmation and assessments. This is shown in Fig. 4.

\subsection{Patterns of fittedness between question and response}

While the grammatical and information structural properties of questions have received widespread attention in linguistics literature, there has been relatively little attention paid to the relationship between questions and their responses. (Note that a full quarter of questions received no response $(78 / 308=25 \%$ ); this includes situations in which Speaker A asks a question, and then follows on talking before Speaker B responds.) Following sections deal with ways in which questions can be responded to: first, looking at content questions, then polar questions.

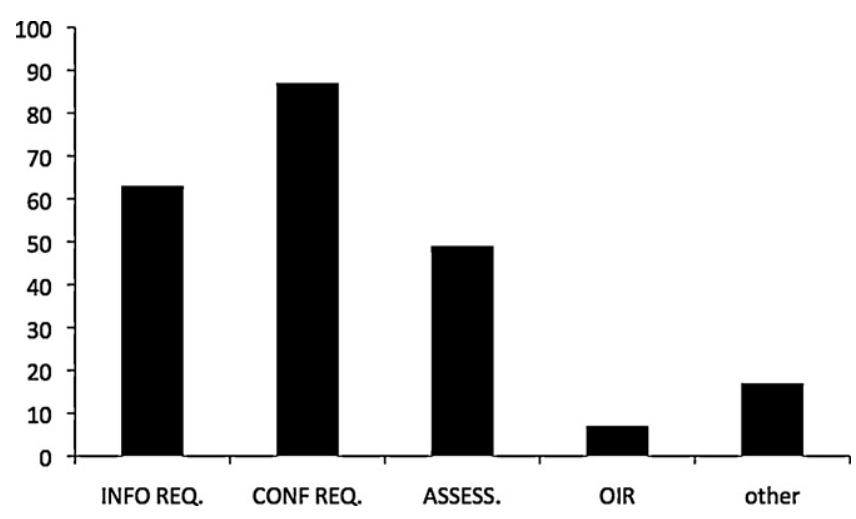

Fig. 4. Frequencies (as $\mathrm{n} / 223$ ) of different functions across polar questions. 


\subsubsection{Responses to content questions}

As noted above, content questions presuppose the truth of some proposition, while narrowing in on some component of the proposition which is unknown. So, Who washed the dishes? presupposes the truth of 'Someone washed the dishes', and focuses on the unknown identity of this someone. Presumably, a maximally conforming response will supply precisely this unknown, sought after piece of information. Example (32) illustrates, with a 'Who?' question.

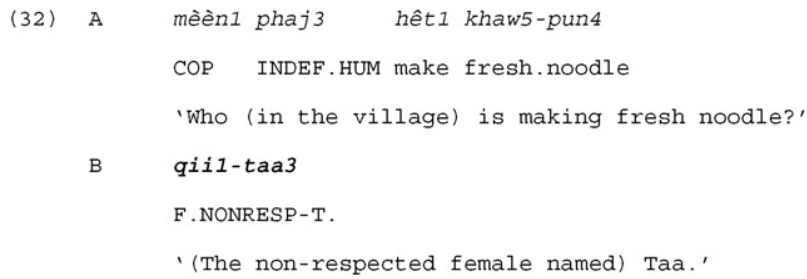

Another example of simple information supply in a response is shown in (33), in which Speaker A's question is responded to by Speaker B and Speaker C in unison.

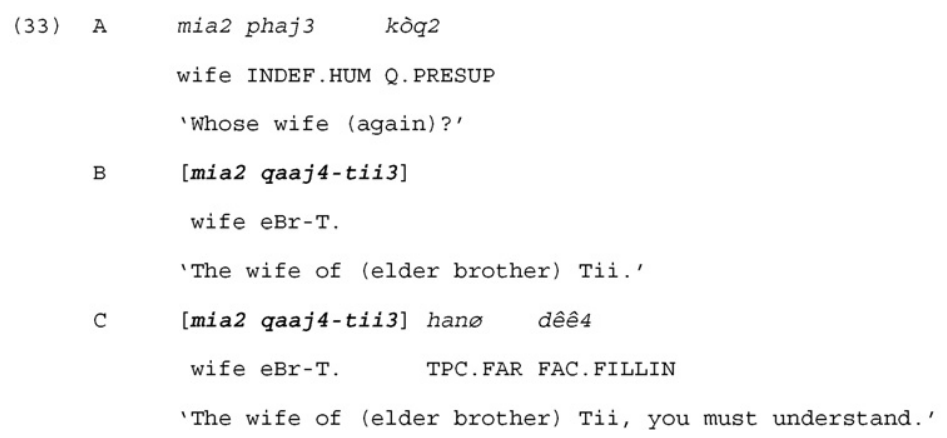

Examples (32) and (33) show responses that directly fit the requirements set out by the questions they are responding to. In the ten-language comparative coding study (see introduction to this special issue), these are coded as 'answers', because they supply the information requested. Well over half of the Lao questions received answers ( $179 / 308=58 \%$ ). Answers made up for over three quarters of all responses $(179 / 230=78 \%)$. Other types of case are coded as 'non-answer response', because while they are relevant as responses to the question, they do not actually directly supply the requested information (although they may do so indirectly). Non-answer responses occurred after $17 \%$ of the total questions $(51 / 308)$.

Example (34) is a case of 'non-answer response', also in the domain of 'Who?' questions.

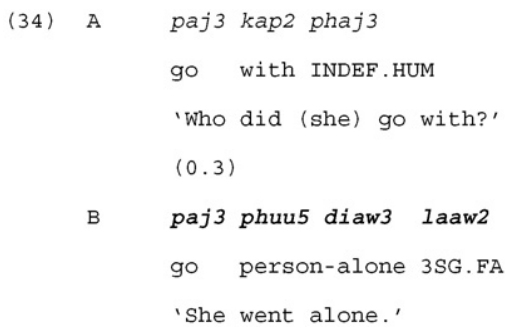

In (34), to be maximally congruent with Speaker A's question, Speaker B would have produced a person reference which identified who it was that 'she' went with. But Speaker B cannot do this, since the presupposition of Speaker A's question is that she indeed did go with someone. As is clear from Speaker B's response, this presupposition is incorrect.

Among content questions, as shown in Fig. 1, above, the most common are those in the 'what' category. These, however, vary in their type of reference. In some cases, the 'what' in question refers to an actual thing (see example (2), above). In other cases, the 'what' in question relates to some feature of a thing, or to the type of thing in question. To respond adequately to a content question, the respondent's formulation needs to pick out just the right category which the question was seeking. In example (35), the referent of 'what?' corresponds to the modifier of the nominal head jaa3 'medicine'. 


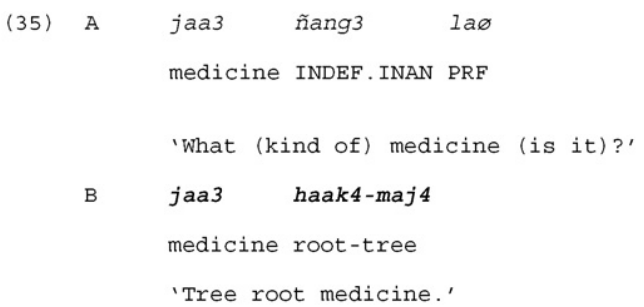

In other cases, the 'what' in question refers not to a thing but to an event, as in What happened? or What did you do?. In example (36), the response properly identifies this event.

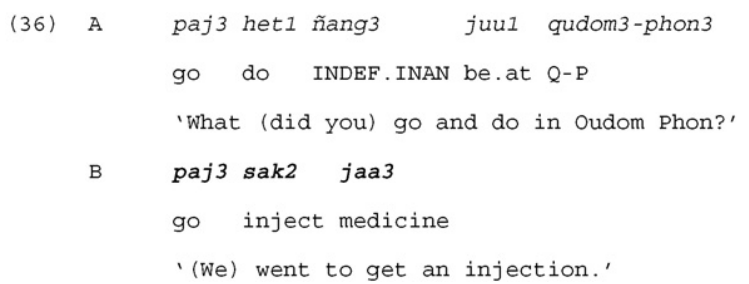

Similarly, content questions focusing on 'where' may be answered in different ways. While we may expect that a conforming answer simply specifies a place, in fact the range of things that may qualify as a place is broad and context-sensitive (Schegloff, 1972). For example, in (37), Speaker A asks as where a certain woman (the current topic of conversation) is living. The response could have been a place name, but the alternative given here is linked to a person (the woman's husband). (Cf. Stivers, 2007 on similar strategies in reference to persons.)

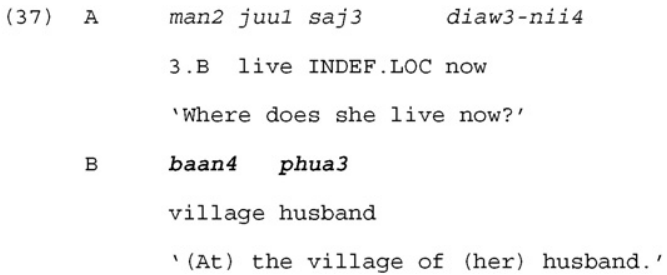

Another way to answer a 'where' question is to focus not on the locational setting of an event or state of affairs, but on the locus of some action within a scene. For example, in a conversation about a young child who has been to hospital for an operation, Speaker A asks where the child was operated on-that is, on what part of his body. This is shown in example (38).

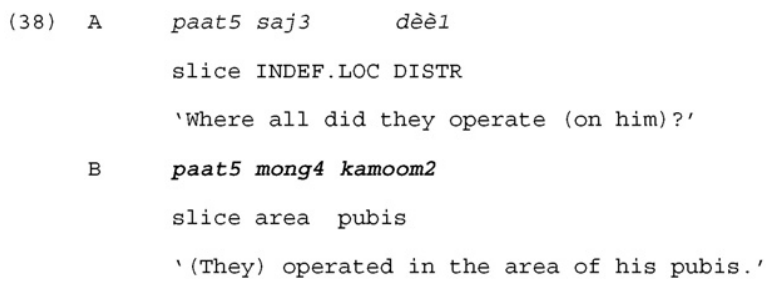

While example (38) focuses in on a second sense of 'where', there are ways to answer a 'where' question which do not mention location at all. In example (39), Speaker A asks where a certain person is. Speaker B's answer does not supply a location, but an activity. The location associated with this activity is known from the common ground of these interlocutors; i.e., they know that a group of villagers has gone to a certain location to collect reeds.

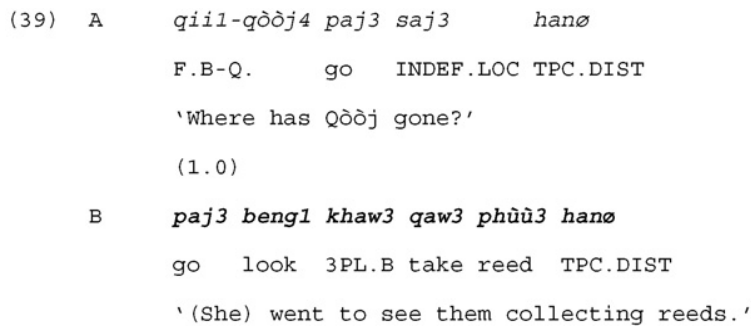


Limitations of space prevent further discussion of the nature of structural fit between content questions and their responses. The above examples from the domains of person, thing and place illustrate the nature of this domain, and the kinds of analytical problems which will in due course need solving. The working hypothesis is that for a response to a content question to be maximally fitted, it should supply a referent of the relevant ontological category (i.e., a thing for a 'what' question, a person for a 'who' question, etc.). Two issues will have to be ironed out. First, each of these ontological categories shows a range of the possible things that may count as instances. For example, a 'what' question can be properly answered with an object or an event (cf. examples (35-36), above). Second, within those things that might count as an appropriate answer (i.e. one that supplies the asked-for referent and accepts the presuppositions inherent in the question), there needs to be a theory of what kinds of utterance will in fact count as an adequate instance of the referential category in question. This issue has been explored in the domain of person reference in Enfield and Stivers (2007). A proper analysis of the system for responding to content questions requires a general theory of referential formulation (or 'word selection' as Schegloff, 2006 puts it), for all the main categories: person reference, place reference, object reference, event reference, time reference, reason reference, and so on.

\subsubsection{Responses to polar questions}

The matter of responding to polar questions is very much simpler in logical terms than the case of content questions described in the previous section. Recall that a polar question effectively puts forward a candidate proposition and asks as to whether this proposition is true or not. The logical answers which can be given are 'yes' and 'no'. Accordingly, many languages have words like English yes and no, dedicated to this function. Another strategy, surely available in every language, is to respond to a polar question by repeating some or all of what was in the question, with negation if applicable. So, possible answers to Is John married to Mary? include Yes, He is, John is married to Mary, No, and He isn't. Each of these options has the function of confirming or disconfirming the truth of the proposition 'John is married to Mary', but in different ways (see discussion in introduction to this special issue; cf. Raymond, 2003).

Lao has more than one type of interjection for saying 'yes'. The most common is qee5, an informal interjection with several functional equivalents, including qùù 5 and $m m 5$. In addition, there are polite forms for confirming polar questions, used in formal situations such as when speaking with a monk, school teacher, or government official. These are caaw4 and dooj3. Because the recordings on which the present research is based are highly informal, there are no instances in this conversational corpus of formal, polite forms.

There are no corresponding polite interjections meaning 'no'. The usual word for 'no' is bòò1. It may be used as a stand-alone response to a polar question, or may be used as a prefixed negator in a more complex construction, bound to the verb or adjective it operates upon. If one really wanted to mark a 'no' response as especially polite, for example when in conversation with an abbot, one might add the word khanò̀j5, literally 'little slave' (a downgrading reference to oneself); as bòò1 khanòò5, something along the lines of No, Sir. Another form of 'no' as a response to a polar question is mq2. This only occurs as a standalone response meaning 'no', and has a minimizing or dismissive tone much like English nope. Its less effortful articulation (one doesn't even have to open one's mouth) is somehow fitting with the minimal nature of its contribution (Gardner, 2001).

Apart from these vocal resources, there are visible ways of saying 'yes' and 'no'. The main visible form for 'yes' is the 'head toss', a single, sharp upwards movement of the head. There is also something more similar to head nods by speakers of English (Stivers, 2008). To say 'no', speakers also sometimes shake their head from side to side. 1 out of 5 responses in the corpus included a visible component such as head toss or a nod (47/230 $=20 \%)$. Most of these occurred together with speech (an example is (27), above). Only 10 responses consisted of a visible component alone (an example is (26), above).

We now turn to some examples of interjection type answers. Example (40) illustrates qee5 'yes' in response to a question which seeks confirmation. In the first line of this example, Speaker A asks a content question, focusing on who has been to collect bamboo shoots. Speaker B does not immediately answer this question, but responds with another question, seeking clarification that Speaker A is talking about the bamboo shoots in a sack which is visible to the two speakers, leaning against the outside wall of a nearby house. Speaker A confirms with qee5 (in bold face below), after which Speaker B is then able to answer the question first posed by A.

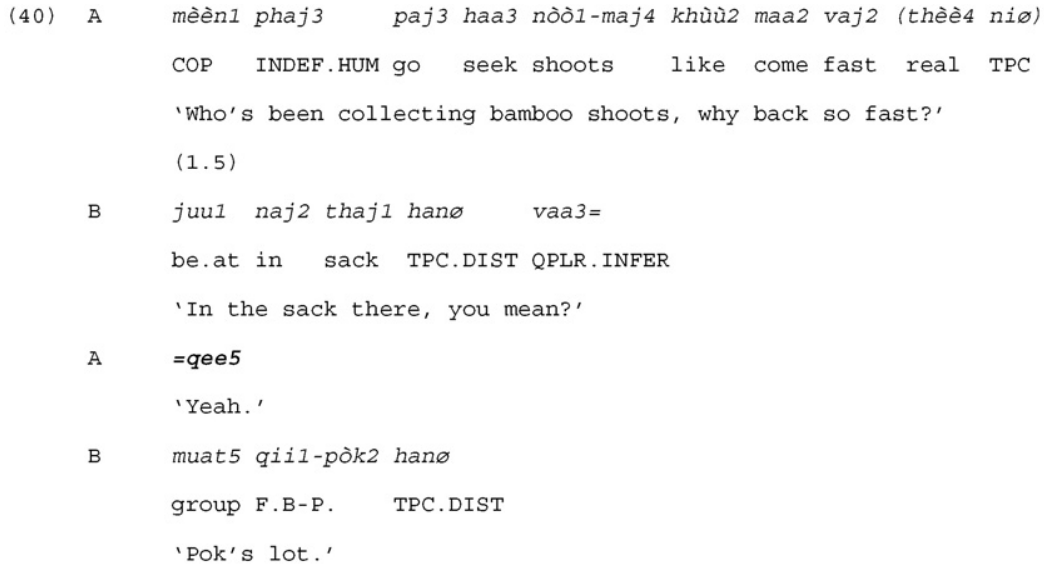


Next is an example of bòò ' 'no' as a disconfirming answer to a polar question. Speaker A is inspecting some herbal medicine he has just been given by Speaker B. In (41), he asks B whether it is in fact a herb by the name of khiing khaj ton. Speaker B disconfirms using the negator bòò as an interjection (in bold face below).

(41) A bòø mèèn khiing3-khaj2-ton4 bòò3
NEG COP K-K-T QPLR
'It isn't khiing khaj ton?'
(0.2)
B bòò1
NEG
'No.'

An alternative to using an interjection in answering a polar question is to repeat part or all of the question. Such repetitional answers are somewhat less frequent in Lao than interjections. Fewer than 1 in 5 responses to polar questions involved repetition $(23 / 128=18 \%)$. I now supply examples of repetitional answers, showing both full and partial repeat (though I am so far unable to identify functional differences between full and partial repetition).

Example (42) shows repetition of an element of the question as a way of confirming. Speakers A and B are gossiping about a new colleague of B's. A asks whether the woman's figure is beautiful. Speaker B, after cutting off her speech which overlapped with A's question (B was apparently going to say something about the woman's hair), answers the question affirmatively, not with qee5 'yes', but with repetition of part of the proposition in question, hun1 ngaam2 '(her) figure is beautiful'.

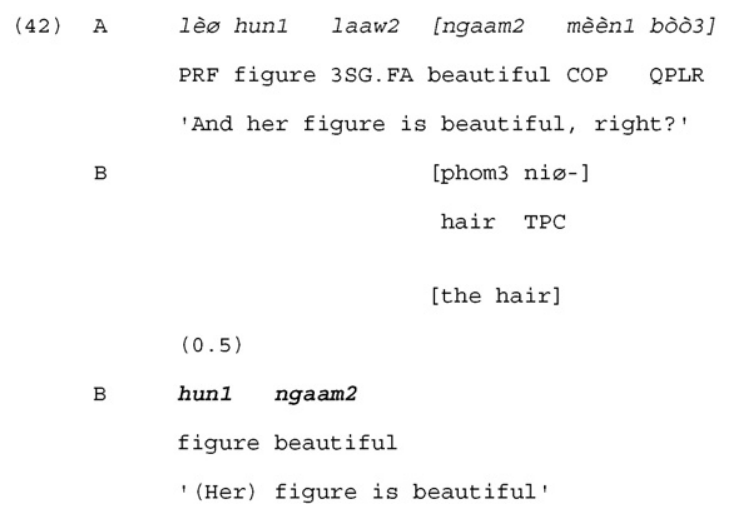

Example (43) also shows confirmation of the question by means of full repetition. Speaker B has been telling a story about a young boy who had to undergo lower abdominal surgery. She has mentioned that the child's penis was also operated on. In (43), Speaker A asks for confirmation, but this is more than merely checking the truth of what Speaker B had said. Perhaps more importantly it is a way for Speaker A to express astonishment. This is also evident in Speaker A's use of the 'far distal' topic marker phunø, conveying the notion of extremeness. Speaker B's confirmation in the second line of the example repeats the content of Speaker A's question.

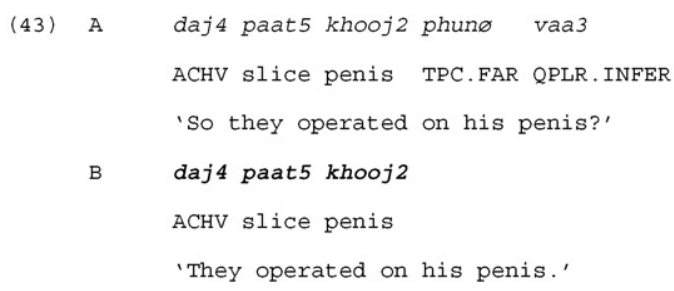

I presume that there is a functional difference in the distinction between responding to a polar question with an interjection versus a repetitional structure (cf. Raymond, 2003 for English). Without further, systematic analysis of a larger set of examples, I am unable to say what that distinction is. This awaits further research.

One way to attenuate the strength of an answer to a polar question is to add an appropriate particle, such as the weak factive particle juu1, as in example (23), repeated from above. 
The following example shows a related manner of producing a response which does not merely say 'yes' or 'no', but adds nuance to the respondent's stance toward the proposition in question. Speakers A and B are chatting in their home village, just after Speaker B has arrived from the city in a pickup truck. Speaker A has asked whether Speaker B brought a man called Loy with him. Loy, a local of the same village, and the son of Speaker B, had been in the city for several days already. Speaker B explains that when he left the city, Loy was there, but he didn't join them on their ride back to the village. Example (44) begins with Speaker A proposing a candidate reason for Loy's not coming, in the form of a 'declarative question'. Speaker B's disconfirming response to this (in bold face in the example) includes not only a negated repetition of the verb khaa2 'to be stuck, busy with something', but also some further modification, including the addition of the topic linker kaø and the perfective particle lèq1 (see Enfield, 2007 for explication of these markers and their meanings). This is followed by two further lines spelling out a strong resistance to the possibility raised in A's question, that Loy may have had some business in the city. In fact, Loy is just goofing off. It turns out that Speaker B, Loy's father, is conveying his annoyance with Loy for shirking his duties in the village.

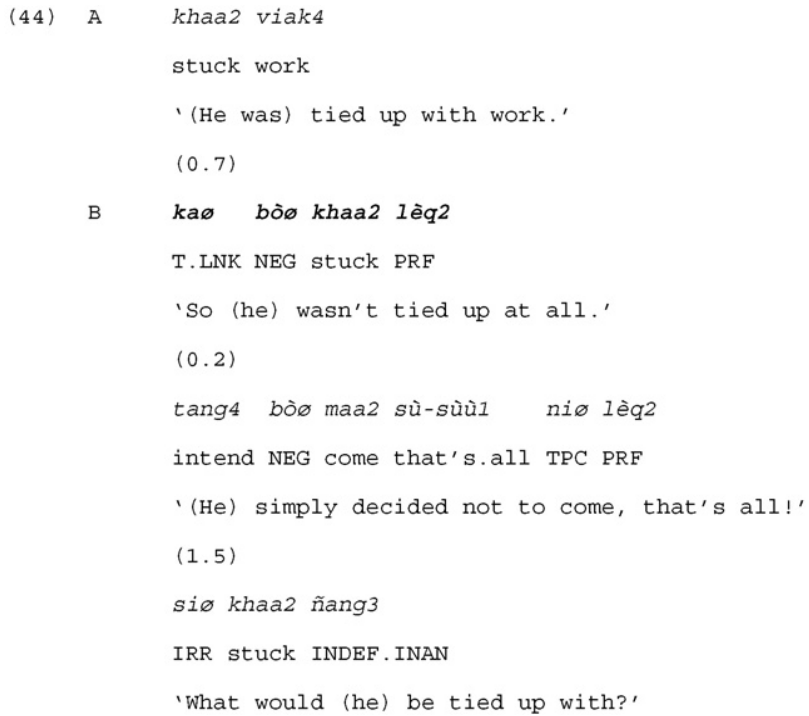

In some cases, an interjection answer is combined with a repetitional answer. In the few cases I have observed, the interjection precedes the repetitional component. This is illustrated in examples (45) and (46).

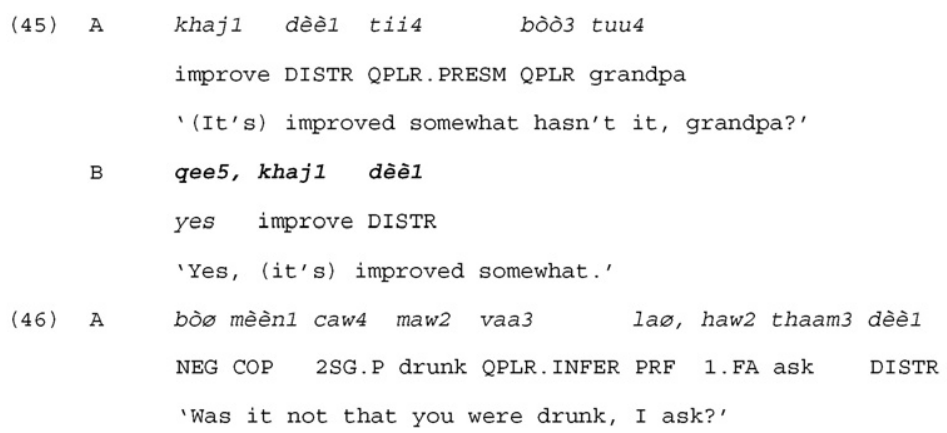




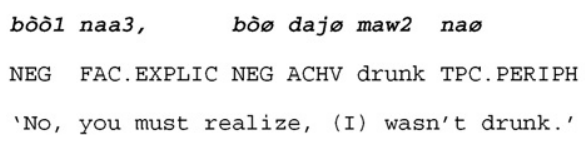

Finally, there are cases in which responses do not directly conform with the terms of the question, but they nevertheless indirectly provide an answer, and supply more information in addition. For example, in (47), Speaker A has seen a pickup truck delivering rice in the village. She asks Speaker B whether the delivery is for Taa, a woman living in a neighboring house. The response from Speaker B (in bold face) does not directly disconfirm (i.e., with an interjection), but effectively disconfirms by means of providing the correct information (namely that they are delivering it to someone else, therefore not to Taa).

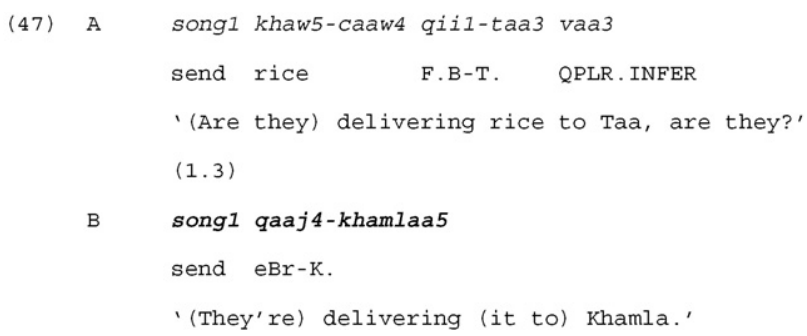

Example (48) shows this strategy as a follow-up to an explicit disconfirmation with $m q 2$ 'nope'. First the respondent disconfirms ('Nope, he wasn't a Lao person'), and secondly, states that the person was Japanese (in itself entailing that he wasn't Lao).

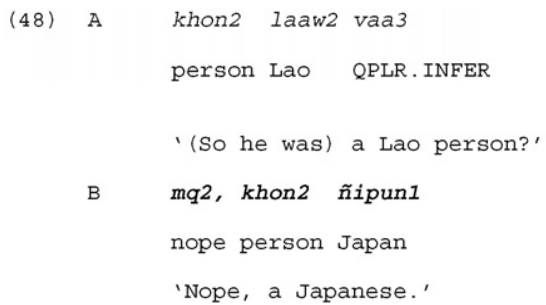

\section{Concluding remarks}

In this paper I have sketched resources in the Lao language not only for asking questions, but also for responding to them. The kinds of variation in the form of questions outlined above is more or less what we might expect from current knowledge in linguistic typology. Less well understood cross-linguistically are the systems of response types. Do these form grammatical systems? The data suggest that they do. The set of options for forms meaning 'yes' in Lao looks not unlike the kind of closed set we might find in a grammatical system like the set of demonstratives or the set of personal pronouns. Choices of forms within such a system contrast with each other, and may interact pragmatically. Within answers, there is an opposition between interjections and repetitional answers as two broad types. Within interjections, there are unequal alternatives (e.g., informal versus polite, spoken versus visible actions like nods and 'head tosses'). (See Enfield, 2009, p67 and passim for a similar situation with demonstratives.) And these as a set contrast with repetitional answers, which, it seems, can vary much more. As a type of polar question response, the repetitional answer is an alternative to the interjection type. Content questions are a very different phenomenon from polar questions in informational terms, and accordingly, the systems for answering these two types of questions are different. The domain of response to content questions appears to be systematic, but less is known. To really figure out the systems for responding to content questions we will need a stock of theories of reference in multiple ontological and conceptual domains: a theory of person reference for answers to 'who' questions, a theory of place reference for answers to 'where' questions, a theory of reason reference for answers to 'why' questions, and so on.

In sum, the range of ways in which a person may respond to questions is not a smooth field of variation. Questions are a way of heavily constraining your interlocutor's next move. Accordingly, grammatical systems reflect this constrainedness.

\section{Acknowledgements}

This work was carried out within the Multimodal Interaction project of the Max Planck Institute for Psycholinguistics, Nijmegen. Many thanks to anonymous reviewers for helpful suggestions for improvement, and to Tanya Stivers for help in developing and clarifying many points. 
Appendix A. Data sources

\begin{tabular}{|c|c|c|}
\hline Media name & Length & Description \\
\hline \multicolumn{3}{|l|}{ Dyadic } \\
\hline 030806a & $16 \min$ & 2 men, neighbors, 40 years and 60 years, in home village \\
\hline 010707 & $10 \mathrm{~min}$ & 2 women, friends, each 25 years, in hotel room \\
\hline 030806e & $6 \mathrm{~min}$ & 2 men, neighbors, 40 years and 75 years, in home village \\
\hline \multicolumn{3}{|l|}{ Multi-party } \\
\hline 020727a & $8 \mathrm{~min}$ & An old couple visiting another old couple's home \\
\hline 15Aug0501 & $11 \mathrm{~min}$ & A father and his son and daughter, in home village \\
\hline 030806b & $10 \mathrm{~min}$ & 3 men, neighbors, 40 years, 60 years, 75 years, in home village \\
\hline 15Aug0503 & $11 \mathrm{~min}$ & 3 women, neighbors, 20 years, 30 years, 40 years, at home \\
\hline 030806k & $6 \min$ & 5 women, neighbors, $20-50$ years, in home village \\
\hline Total & $78 \mathrm{~min}$ & \\
\hline
\end{tabular}

\section{References}

Crisfield, Arthur G., 1974. Lao final particles. In: Nguyen, Dang Liem (Ed.), Southeast Asian Linguistic Studies, vol. 1. Pacific Linguistics, Canberra, pp. 41-45. Enfield, N.J., 2007. A Grammar of Lao. Mouton de Gruyter, Berlin.

Enfield, N.J., 2009. The Anatomy of Meaning: Speech, Gesture, and Composite Utterances. Cambridge University Press, Cambridge.

Enfield, N.J., Stivers, Tanya (Eds.), 2007. Person Reference in Interaction: Linguistic, Cultural, and Social Perspectives. Cambridge University Press, Cambridge.

Gardner, Rod, 2001. When Listeners Talk: Response Tokens and Listener Space. John Benjamins, Amsterdam.

Grice, H.Paul, 1975. Logic and conversation. In: Cole, Peter, Morgan, Jerry L. (Eds.), Speech Acts. Academic Press, New York, pp. 41-58.

Levinson, Stephen C., 1983. Pragmatics. Cambridge University Press, Cambridge.

Levinson, Stephen C., 2000. Presumptive Meanings: The Theory of Generalized Conversational Implicature. MIT Press, Cambridge, MA/London.

Raymond, Geoffrey, 2003. Grammar and social organization: yes/no interrogatives and the structure of responding. American Sociological Review 68, 939-967.

Schegloff, Emanuel A., 1972. Notes on a conversational practice: formulating place. In: Sudnow, David (Ed.), Studies in Social Interaction. The Free Press, New York, pp. 75-119.

Schegloff, Emanuel A., 2006. Interaction: the infrastructure for social institutions, the natural ecological niche for language, and the arena in which culture is enacted. In: Enfield, N.J., Levinson, Stephen C. (Eds.), Roots of Human Sociality: Culture, Cognition, and Interaction. Berg, Oxford, pp. 70-96.

Searle, John R., 1969. Speech Acts: An Essay in the Philosophy of Language. Cambridge University Press, Cambridge.

Sperber, Dan, Wilson, Dierdre, 1995. Relevance: Communication and Cognition, 2nd edition. Blackwell, Oxford.

Stivers, Tanya, 2007. Alternative recognitionals in person reference. In: Enfield, N.J., Stivers, Tanya (Eds.), Person Reference in Interaction: Linguistic, Cultural, and Social Perspectives. Cambridge University Press, Cambridge, pp. 73-96.

Stivers, Tanya, 2008. Stance, alignment and affiliation during story telling: when nodding is a token of preliminary affiliation. Research on Language and Social Interaction 41 .

Wierzbicka, Anna, 1980. Lingua Mentalis. Academic Press, Sydney.

Wierzbicka, Anna, 1996. Semantics: Primes and Universals. Oxford University Press, Oxford.

N.J. Enfield is a senior staff scientist at the Max Planck Institute for Psycholinguistics, and is Professor of Ethnolinguistics at Radboud University Nijmegen. He is leader of the European Research Council project 'Human Sociality and Systems of Language Use'. His research on semiotic systems is based on regular fieldwork in mainland Southeast Asia. Publications include Linguistic Epidemiology (2003, Routledge), A Grammar of Lao (2007, Mouton), and The Anatomy of Meaning (2009, Cambridge), and edited volumes Ethnosyntax (2002, Oxford), Roots of Human Sociality (2006, Berg, with SC Levinson), and Person Reference in Interaction (2007, Cambridge, with T Stivers). 